\title{
Effect of central and peripheral cone- and rod-specific stimulation on the pupillary light reflex
}

\author{
Anton Sonntag • Carina Kelbsch • Ronja Jung • Helmut Wilhelm • \\ Torsten Strasser $\cdot$ Tobias Peters $\cdot$ Krunoslav Stingl (1) B Barbara Wilhelm
}

Received: 14 June 2021/Accepted: 12 November 2021 / Published online: 26 November 2021

(C) The Author(s) 2021

\begin{abstract}
Purpose To assess the effect of central and peripheral stimulation on the pupillary light reflex. The aim was to detect possible differences between cone- and rod-driven reactions.

Methods Relative maximal pupil constriction amplitude (relMCA) and latency to constriction onset (latency) to cone- and rod-specific stimuli of 30 healthy participants $(24 \pm 5$ years (standard deviation)) were measured using chromatic pupil
\end{abstract}

Supplementary Information The online version contains supplementary material available at https://doi.org/10.1007/ s10792-021-02132-1.

A. Sonntag $\cdot$ C. Kelbsch $\cdot$ R. Jung .

H. Wilhelm - T. Strasser - T. Peters .

K. Stingl $(\square) \cdot$ B. Wilhelm

Pupil Research Group, Centre for Ophthalmology,

University of Tübingen, Elfriede-Aulhorn-Str. 7,

72076 Tübingen, Germany

e-mail: krunoslav.stingl@med.uni-tuebingen.de

C. Kelbsch · R. Jung $\cdot$ H. Wilhelm •

T. Strasser $\cdot$ K. Stingl

University Eye Hospital, Centre for Ophthalmology,

University of Tübingen, Tübingen, Germany

T. Strasser

Institute for Ophthalmic Research, Centre for

Ophthalmology, University of Tübingen, Tübingen,

Germany campimetry. Cone- and rod-specific stimuli had different intensities and wavelengths according to the Standards in Pupillography. Five filled circles with radii of $3^{\circ}, 5^{\circ}, 10^{\circ}, 20^{\circ}$ and $40^{\circ}$ and four rings with a constant outer radius of $40^{\circ}$ and inner radii of $3^{\circ}, 5^{\circ}$, $10^{\circ}$ and $20^{\circ}$ were used as stimuli.

Results For cone-and rod-specific stimuli, relMCA increased with the stimulus area for both, circles and rings. However, increasing the area of a cone-specific ring by minimizing its inner radius with constant outer radius increased relMCA significantly stronger than the same did for a rod-specific ring. For cones and rods, a circle stimulus with a radius of $40^{\circ}$ created a

\footnotetext{
K. Stingl

Centre for Rare Eye Diseases, University of Tübingen, Tübingen, Germany
} 
lower relMCA than the summation of the relMCAs to the corresponding ring and circle stimuli which combined create a $40^{\circ}$ circle-stimulus. Latency was longer for rods than for cones. It decreased with increasing stimulus area for circle stimuli while it stayed nearly constant with increasing ring stimulus area for cone- and rod-specific stimuli.

Conclusion The effect of central stimulation on relMCA is more dominant for cone-specific stimuli than for rod-specific stimuli while latency dynamics are similar for both conditions.

Keywords Pupil campimetry · Pupillography · Cones $\cdot$ Rods $\cdot$ Centre $\cdot$ Periphery

\section{Introduction}

Pupil campimetry, full-field pupillography and multifocal pupil perimetry are increasingly being employed in the clinic and are under further investigation by several research groups as methods to detect retinal and optic neuropathies such as glaucoma, age-related macular degeneration, retinitis pigmentosa and diabetic retinopathy in type 2 diabetes [1-11].

The use of specific wavelengths, stimulus durations and adaptation states at different locations of the visual field allows responses from rods, cones and intrinsically photosensitive retinal ganglion cells to be studied by pupil campimetry [12]. Based on several studies using chromatic pupillography that detected changes of the pupillary light reflex (PLR) to explicitly celladdressing stimuli in patients with retinal diseases $[13,14]$ and glaucoma [15] the Pupil Research Group at the Centre for Ophthalmology developed a new type of device, the Chromatic Pupil Campimeter (CPC): a combination of cell-specific stimuli and a new pupillographic campimetry device introduced by Stingl et al. [1]. Kelbsch et al. presented this objective method to measure pupil responses separately for rods and L-cones at different locations in the visual field [2] and found that the mean relative maximal constriction amplitude (relMCA) caused by cone-specific stimuli was larger in the centre of the retina and decreased in a hill-shaped form towards the periphery. Rod-specific relMCAs were smaller and showed a flatter profile around the retina with only a minor peak in the centre. Patients with rod or cone deficiencies showed no rod- and severely impaired cone-specific pupillary reactions respectively [2].

Despite the arising use of pupillography still only little is known about the characteristics of pupillomotor receptive fields, especially those of photoreceptorspecific stimulation.

Skorkovska et al. were the first to explicitly examine summation effects within the pupillary pathway using white light and found that pupillomotor receptive fields are larger than receptive fields for visual perception, that their size increases with increasing retinal eccentricity and that their size decreases with increasing brightness [16].

It is well-known that the amplitude of the PLR is linearly related to the logarithm of the stimulus intensity [17-19]. The light-adapted pupil size depends on the product of stimulus luminance and area (corneal flux density, CFD) [20, 21]. Hu et al. found a correlation between the maximum constriction amplitude, eccentricity and CFD when using white peripheral stimuli [22]. In contrast, Park et al. investigated the relationship between the CFD and the PLR using central stimuli and found that only rod- and melanopsin-mediated pupillary responses were CFDdependent. On the other hand, they found that conemediated responses depended only on stimulus luminance and not on stimulus size [23].

Previous studies have shown an eccentricity effect on the PLR to local stimuli with a decrease from the centre to the periphery for white light $[1,16,19,22,24]$ and also for dim blue and red light under rod- and cone-specific conditions [2, 25]. The eccentricity effect in these studies was larger for conespecific stimulation than for rod-specific stimulation. However, Joyce et al. did not observe a difference in the relMCA for corneal flux density equated photopic short- and long-wavelength stimuli presented at $0^{\circ}$ and $20^{\circ}$ eccentricity [26].

Regarding this limited state of scientific knowledge about summation effects and the observed eccentricity effect on the PLR, we wanted to gain more insight into this topic, particularly on the effect of central versus peripheral stimulation, which is relevant for clinical use of pupillography. Our study aimed to assess the effect of central and peripheral cone- and rod-specific stimulation on the pupillary light reflex in a clinically applicable set-up and to detect differences between cone and rod influence using CPC $[1,2]$. 


\section{Methods}

Participants and ethical aspects

The inclusion criteria were: healthy participants between 18 and 50 years, no ocular disease, no medication that could affect the pupillary light reflex and a refraction error less than or equal to $\pm 3 \mathrm{D}$. To exclude any pathological findings, each participant underwent a medical anamnesis and an ophthalmological examination at the neuro-ophthalmology unit of the University Eye Hospital Tübingen, including visual acuity test, swinging-flashlight-test, slit-lampexamination and funduscopy, optical coherence tomography (OCT) (Spectralis-OCT; Heidelberg Engineering $\mathrm{GmbH}$, Germany) and $30^{\circ}$ visual field examination (static automated strategy, Octopus 101 or 900; Haag-Streit International, Wedel, Germany). Thirty healthy volunteers (14 males, 16 females) with a mean age of $24 \pm 5$ years (standard deviation) were enrolled in the study. They were recruited either from the staff of the University Eye Hospital Tübingen or from the student body of the University of Tübingen. All participants received detailed information about the study and its aims and gave their written informed consent. The study was approved by the local institutional ethics committee (project-number: $775 / 2016 \mathrm{BO} 2$ ) and obeyed the tenets of the Declaration of Helsinki.

\section{Chromatic pupil campimetry set-up}

A modified version of the CPC as described by Kelbsch et al. [2] was used to specifically stimulate predominantly rods or cones, respectively. The experiment was performed in a completely dark and quiet room of the University Eye Hospital and was about one hour in duration. The participants sat on a comfortable chair in front of an OLED-monitor (LG OLED 55C7V), located at a distance of $40 \mathrm{~cm}$ in front of the subject's eye and presenting the stimuli (see Fig. 1). The head was placed in a combined chin- and headrest for stabilization and comfort. Only the left eye was examined while the right eye was covered by an eyepatch. An infrared camera (DMK23UV024, The Imaging Source $\mathrm{GmbH}$ ) with a temporal resolution of $10 \mathrm{~ms}$ and a 50-mm TV-lens 1:1.4, located below the screen, recorded the pupil continuously. The pupil diameter was calculated online by a Java ${ }^{\mathrm{TM}^{2}}$-based in-

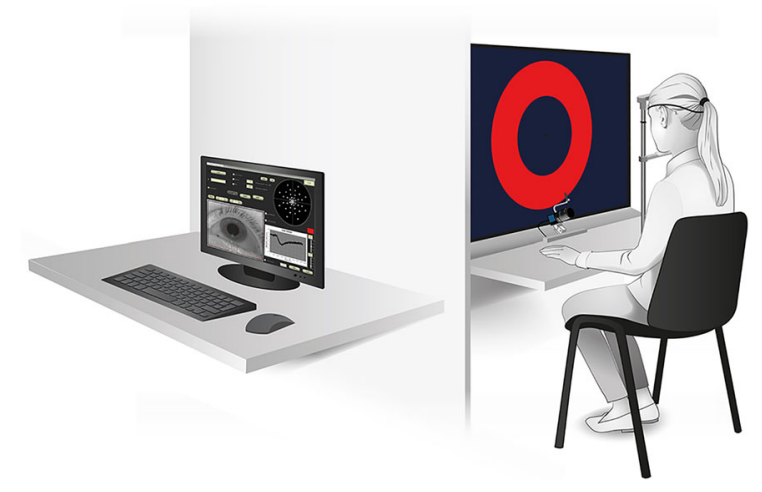

Fig. 1 The setup of the examinations: participants sat on a comfortable chair and stimuli were presented on a large monitor while the examiner sat in front of a darkened computer monitor behind a partition wall

house software described by Stingl et al. [1] using a modified Starburst algorithm [27] that detected the black pupil's edge from the image captured by the camera. An ellipse was fitted to the points of the detected edge using a random sample consensus (RANSAC) approach [28] and the pupil centre and diameter were determined in real-time [1]. The estimated error of the algorithm was approximately 1-2 pixels which correspond to the estimated error of $0.1 \mathrm{~mm}$ per image. A dim fixation point $\left(0.01 \mathrm{~cd} / \mathrm{m}^{2}\right)$ with a radius of $1^{\circ}$ was presented in the centre of the OLED screen.

Stimulus characteristics

Stimuli were created by an in-house developed software as described by Stingl et al. [1]. We used cone- and rod-specific stimuli whose intensity was modified according to Kelbsch et al. [2]. The intention was not to drive the M. sphincter pupillae to its mechanical limits despite a relatively large stimulus size. L-cone-specific stimuli (photCPC) had a luminance of $4 \mathrm{~cd} / \mathrm{m}^{2}$ and a wavelength of $620 \pm 30 \mathrm{~nm}$ full width at half maximum (FWHM). Stimulus duration was $1000 \mathrm{~ms}$ and stimuli were presented on a dim blue background with a luminance of $0.01 \mathrm{~cd} /$ $\mathrm{m}^{2}$ and a wavelength of $460 \pm 30 \mathrm{~nm}$ FWHM. The test was applied after $10 \mathrm{~min}$ of light adaptation to the background to partially saturate the rods [29]. Rodspecific stimuli (scotCPC) had a luminance of $0.001 \mathrm{~cd} / \mathrm{m}^{2}$ and a wavelength of $460 \pm 30 \mathrm{~nm}$ FWHM. Their stimulus duration was $100 \mathrm{~ms}$ and they 
were presented on a completely dark background $\left(0 \mathrm{~cd} / \mathrm{m}^{2}\right)$ after $20 \mathrm{~min}$ of dark adaptation.

The energy and spectrum of our stimuli were measured at the position of the proband's eye using an LED-Spectrometer (MK-350S-Premium, UPRTek, Taiwan). The results are shown in supplementary Fig. S1. Luminance, spectrum and duration of our stimuli are in accordance with the ISCEV Standard for (cone- and rod-specific) full-field clinical electroretinography [30] as well as the Standards in Pupillography [29]. The background during and before the application of cone-specific stimuli was used to partially saturate rods.

The baseline period before the first stimulus presentation for all stimuli was $500 \mathrm{~ms}$ and the interstimulus interval was $4500 \mathrm{~ms}$. The baseline pupil size was calculated for each step individually. A stimulus was repeated automatically if a blink occurred during the presentation or if at least $90 \%$ of the initial pupil diameter was not reached before the following stimulus presentation.

There were 9 different stimuli for either rods or cones and each one was repeated 10 times. Stimuli consisted of five filled circles with a radius of $3^{\circ}, 5^{\circ}$, $10^{\circ}, 20^{\circ}$ and $40^{\circ}$ and four rings with a constant outer radius of $40^{\circ}$ and inner radii of $3^{\circ}, 5^{\circ}, 10^{\circ}$ and $20^{\circ}$ (see Fig. 2).

In each protocol the circles were presented first, beginning with 10 repetitions of the smallest circle and ending with 10 repetitions of the largest circle. Subsequently, the rings were presented, starting with the ring with the largest inner radius (smallest stimulated area) and ending with the ring with the smallest inner radius (largest stimulated area).

Table 1 shows the area of the different stimuli in $\mathrm{deg}^{2}$, calculated using the radii in degree and the formula $A=\pi r^{2}$.

Data management and statistical analysis

Mean relative maximal pupillary constriction amplitude (relMCA) and mean latency to constriction onset (latency) to the different stimuli were determined (for more details see supplementary information, Fig. S2). For the further statistical analysis, one participant was excluded because his relative pupillary responses to the rod-specific stimuli lay two standard deviations below those of all the other participants. Data were visually inspected for following a normal distribution. Analyses of variance (ANOVA) for repeated measurements and Bonferroni corrected post-hoc tests (two-tailed paired t-test) were carried out between relMCAs as well as between latencies to the cone and rod-specific circle- and ring-stimuli. Additionally, a two-tailed paired t-test between the rod-and conespecific difference of relMCA between the $20^{\circ} / 40^{\circ}$ and the $3 \% 40^{\circ}$ ring as well as one between the rod- and cone-specific latencies to the stimuli were performed. Furthermore, relMCAs to the corresponding ring and circle stimuli which combined create a $40^{\circ}$ circlestimulus were summed. An ANOVA for repeated measurements and a Bonferroni corrected post-hoc test (two-tailed paired t-test) were subsequently
Fig. 2 Shapes of applied stimuli. This figure shows the red versions (conespecific stimuli, photCPC; scotopic stimuli used blue colour). There were 9 different stimuli for either cones or rods and 10 repetitions. Below the stimuli, the abbreviations are given which are used hereinafter

\section{Circles:}
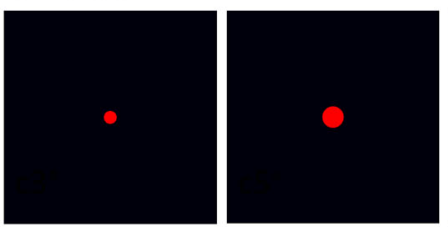
$\mathrm{c}^{\circ}$ c5 ${ }^{\circ}$

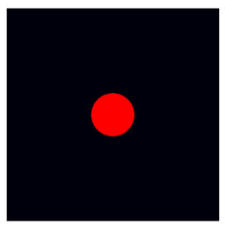

c10 $10^{\circ}$

Rings:
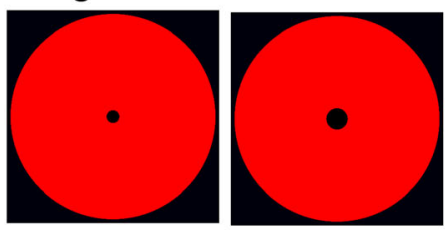

$r 3^{\circ} / 40^{\circ}$

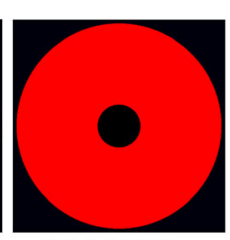

$\mathrm{r} 10^{\circ} / 40^{\circ}$

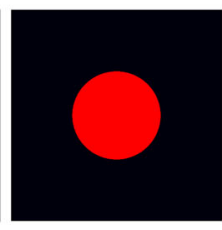

c20 $20^{\circ}$

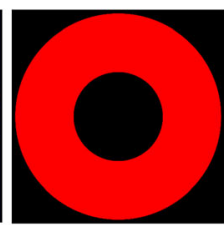

r $20^{\circ} / 40^{\circ}$

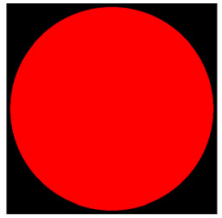

c $40^{\circ}$ 
Table 1 The area of each stimulus in degrees visual angle $^{2}$ (rounded to whole numbers) in phot CPC and scotCPC

\begin{tabular}{ll}
\hline Stimulus & Area $\left(\mathrm{deg}^{2}\right)$ \\
\hline $\mathrm{c} 3^{\circ}$ & 28 \\
$\mathrm{c} 5^{\circ}$ & 79 \\
$\mathrm{c} 10^{\circ}$ & 314 \\
$\mathrm{c} 20^{\circ}$ & 1257 \\
$\mathrm{c} 40^{\circ}$ & 5027 \\
$\mathrm{r} 20^{\circ} / 40^{\circ}$ & 3770 \\
$\mathrm{r} 10^{\circ} / 40^{\circ}$ & 4712 \\
$\mathrm{r} 5^{\circ} / 40^{\circ}$ & 4948 \\
$\mathrm{r} 3 \% / 40^{\circ}$ & 4998 \\
\hline
\end{tabular}

carried out between the different combinations of summation.

RelMCAs and latencies of 29 participants were analysed. $p$-Values smaller than 0.05 were considered statistically significant. Results are presented with mean \pm standard deviation (SD).

\section{Results}

Baseline pupil diameter

The average baseline pupil diameter of the 29 participants during photCPC was $6.3 \pm 1.1 \mathrm{~mm}$ and the average baseline pupil diameter during scotCPC was $7.4 \pm 0.8 \mathrm{~mm}$.

Effect of increasing the stimulus: non-linear increase of pupil response with increasing stimulus area

\section{Cone-specific protocol: photopic CPC (photCPC)}

In Fig. 3 the results of the cone and rod response to circle and ring stimuli are shown. For cones, the relMCA significantly increased with increasing the radius of the circle stimulus $(p<0.001)$. The response to a $3^{\circ}$ circle was $16.2 \pm 6.3 \%$ while the response to a $40^{\circ}$ circle was $33.0 \pm 4.1 \%$. Likewise, the relMCA significantly increased with increasing area of the ring stimulus $(p<0.001)$ : The response to a $20^{\circ} / 40^{\circ}$ ring was $22.4 \pm 5.9 \%$, while the response to a $3 \% 40^{\circ}$ ring was $30.8 \pm 4.6 \%$. The response to the increase of the stimulated area, whether circles or rings, was nonlinear.

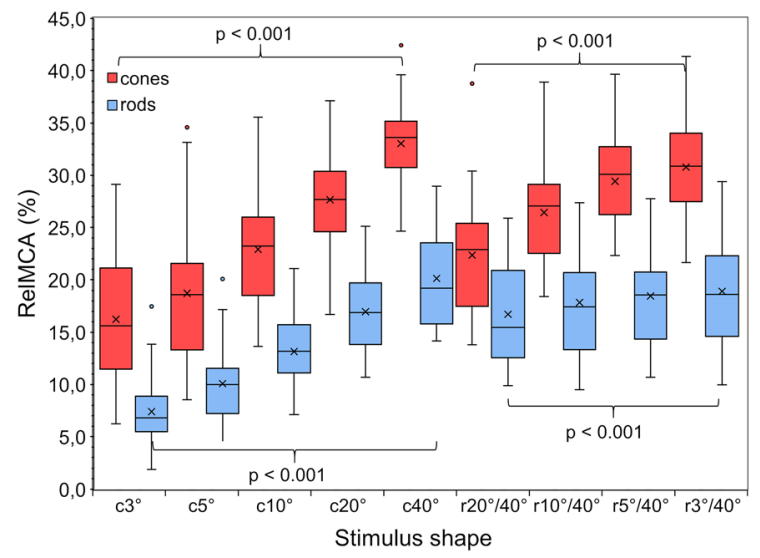

Fig. 3 The average (of 10 pupil responses per participant) relMCAs (relative maximal pupillary constriction amplitudes) to the different stimuli are shown by the box-whisker plots $(n=29)$. The mean values are shown by the crosses. Responses to cone-specific stimuli are coloured red, responses to rodspecific stimuli are coloured blue. The most important significant differences mentioned in the text are shown by the braces. Shapes are explained in Fig. 2

\section{Rod-specific protocol: scotopic CPC (scotCPC)}

The results for the rod-specific protocols (Fig. 3) showed overall smaller relMCAs than those for the photCPC.

As in the photCPC, the relMCA significantly increased with increasing radius of the circle stimulus and there was a significant effect of stimulus size on relMCA $(p<0.001)$. The response to a $3^{\circ}$ circle was $7.4 \pm 3.3 \%$, while the response to a $40^{\circ}$ circle was $20.1 \pm 4.6 \%$. Also, the relMCA significantly increased with increasing area of the ring stimulus, although the difference is only small: The response to a $20^{\circ} / 40^{\circ}$ ring was $16.7 \pm 5.0 \%$ while the response to a $3 \% 40^{\circ}$ ring was $18.9 \pm 5.1 \%$ (for both: $p<0.001$ ). Nevertheless, the increase of the relMCA to rodspecific rings was statistically significant smaller than to cone-specific rings. There was an absolute difference of only $2.2 \pm 1.7 \%$ between the $20^{\circ} / 40^{\circ}$ and the $3 \% / 40^{\circ}$ ring for the rod-specific stimuli. For the conespecific stimuli, the corresponding difference was significantly larger at $8.4 \pm 3.4 \%(p<0.001)$. The difference between the rod-specific relMCAs to the $10^{\circ} / 40^{\circ}$ and the $5^{\circ} / 40^{\circ}$ ring, as well as the difference between the ones to the $5^{\circ} / 40^{\circ}$ and the $3 \% / 40^{\circ}$ ring, were small and non-significant.

The increase of the response to the rod-specific circles and rings was also non-linear. 
Supplementary Fig. S3 shows some exemplary pupil traces of proband number 1 .

For both, cones and rods, a circle stimulus with a radius of $40^{\circ}$ created a significantly smaller relMCA $(p<0.001)$ than the summed response to the ring and circle stimuli which create a $40^{\circ}$ circle stimulus if combined (e. g. $c 3^{\circ}+r 3^{\circ} / 40^{\circ}$ ) (see supplementary Fig. S4).

\section{Latency to constriction onset}

In Fig. 4 we plot the latency to constriction onset for the cone and rod stimuli for the circle and ring stimuli.

\section{PhotCPC: influence of stimulus configuration on latency}

For the cone-specific stimuli (red boxplots in Fig. 4), the mean latency decreased significantly with increasing radius of circle stimulus. The effect of stimulus size on latency was significant $(p<0.001$ for circles, $p=0.004$ for rings). The latency to a $3^{\circ}$ circle was $329 \pm 34 \mathrm{~ms}$ while the latency to a $40^{\circ}$ circle was $254 \pm 28 \mathrm{~ms}(p<0.001)$. In contrast, latency stayed nearly constant with increasing area of the ring stimulus, though the effect of a smaller inner radius was still significant. The latency to a $20^{\circ} / 40^{\circ}$ ring was

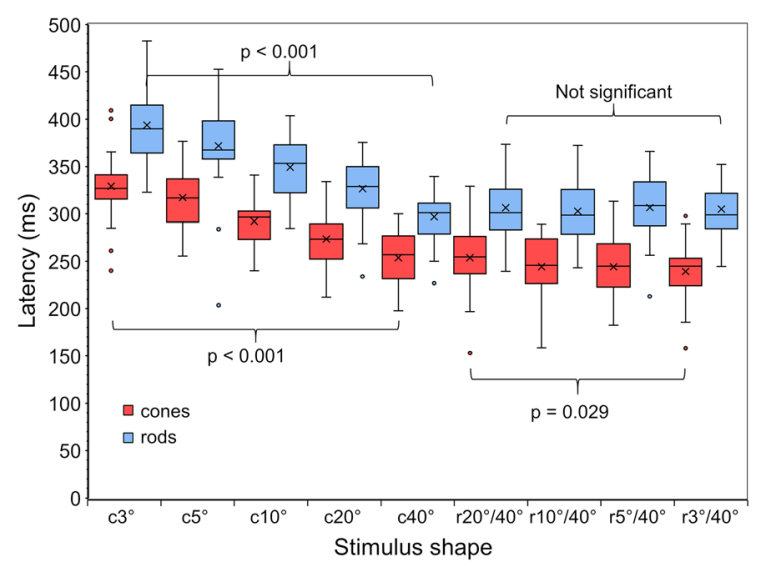

Fig. 4 The average (of 10 pupil responses per subject) latencies to constriction onset to the circle and ring stimuli are shown by the box-whisker plots $(n=29)$. The mean values are shown by the crosses. Latencies to cone-specific stimuli are coloured red, latencies to rod-specific stimuli are coloured blue. The most important significant differences mentioned in the text are shown by the braces
$254 \pm 35 \mathrm{~ms}$ and different from the latency to a $3 \%$ $40^{\circ}$ ring that was $239 \pm 31 \mathrm{~ms}(p=0.029)$.

\section{ScotCPC: longer latencies}

The results for the rod-specific stimuli (blue boxplots in Fig. 4) showed overall longer mean latencies to constriction onset than those to the photCPC $(p<0.001)$. The mean latency difference between rods and cones was $57 \pm 32 \mathrm{~ms}$. As in the photCPC, latency decreased significantly with a larger radius of circle stimuli. The effect of circle stimulus size on latency was significant $(p<0.001)$. The latency to a $3^{\circ}$ circle was $394 \pm 40 \mathrm{~ms}$ while the latency to a $40^{\circ}$ circle was $297 \pm 26 \mathrm{~ms}(p<0.001)$.

In contrast, there was no significant decrease of latency with a larger area of the ring stimulus. The effect of ring stimulus size on latency is not significant. The latency to a $20^{\circ} / 40^{\circ}$ ring was $306 \pm 33 \mathrm{~ms}$ and the latency to a $3 \% 40^{\circ}$ ring was $305 \pm 31 \mathrm{~ms}$ (non-significant).

\section{Discussion}

Our study focuses on the effect of central and peripheral stimulation on the pupillary light reflex. The aim was to detect possible differences between cone- and rod-driven reactions.

For both, cone- and rod-specific stimulation, relMCA increased with increasing stimulus size (see Fig. 3). This was expected as a larger stimulus activates a larger area of the retina. It is well-known that the amplitude of the PLR is linearly related to the logarithm of the stimulus intensity [17-19] but differing results on the relationship between PLR and stimulus size have been reported in the literature. Various studies have shown that the light-adapted pupil size depends on the product of luminance and stimulus area, the corneal flux density (CFD) [20, 21]. $\mathrm{Hu}$ et al. expressed the maximum pupil constriction to white peripheral stimuli as a function of CFD and eccentricity [22]. Joyce et al. examined the relMCA caused by long- and short-wavelength stimuli under conditions constant for either CFD, irradiance or stimulus-size [26]: their study revealed that for both wavelengths a constant CFD resulted in a relatively constant pupillary constriction. A CFD-dependency of the maximum pupil constriction agrees with our 
results as the relMCA of cone- and rod-mediated responses increased with increasing stimulus size and consequently a larger CFD.

Cone-specific stimulation revealed that the effect of central stimulation is dominant for the PLR: although the stimulated area is much larger, the relMCA to a $40^{\circ}$ circle stimulus was only two times stronger than the relMCA to a $3^{\circ}$ circle (see Fig. 3). Moreover, increasing the area of central stimulation while the periphery is also stimulated had a significant effect on the pupillary response as well: Minimizing the inner radius of a ring-stimulus increased the pupillary response amplitude by more than one third.

With rod-specific stimuli, the effect of central stimulation is less dominant for the PLR than with cone-specific stimuli. The relMCA to a $40^{\circ}$ circle stimulus was three times as large as the relMCA to a $3^{\circ}$ circle (versus two times as large in photCPC) (see Fig. 3). Furthermore, in contrast to the photCPC, the increase of amplitude to the increasing size of ring stimuli (smaller inner radius) was significantly smaller than for cone-specific stimuli. Increasing the area of central stimulation while the periphery was already stimulated increased the pupillary response amplitude only minimally. One could argue that this is not surprising because there are almost no rods in the centre of the retina [31], but according to Curcio et al., the size of the average diameter of the rod-free zone in the middle of the retina is only $1.25^{\circ}$ [31] which is also the reason for a response to our $\mathrm{c} 3^{\circ}$-rod-stimulus.

For cones and rods, a circle stimulus with a radius of $40^{\circ}$ created a lower relMCA than the summation of the relMCAs to the corresponding ring and circle stimuli which combined create a $40^{\circ}$ circle-stimulus. This indicates a non-linear summation.

In our recent publication on CPC [2] and a study by Haj Yahia et al. [25] a prominent role of cones in the centre of the retina has also been described, i.e. larger eccentricity effect for cones than for rods. In patients with AMD the relMCAs to local stimuli were lower than those of an age-matched healthy control group, especially for the centre of the visual field. In addition, the profile of responses over the retina was flatter [6]. Our results are in line with these studies, although their results were based on local stimulation. Skorkovska et al. examined summation effects of the pupillary light reflex to white light under conditions of light adaptation and found the amplitude of the PLR being related to size, intensity and retinal location. They observed an eccentricity effect with lower relMCAs in the periphery [16]. However, their results cannot simply be transferred to our photoreceptor-specific results as they used other stimulus shapes, white light and no photoreceptor-specific stimulation. Several other studies have found an effect of eccentricity on the PLR to white light $[1,19,22,24]$.

Regarding latency to constriction onset, it can be seen that latency was significantly shorter for cones than for rods (see Fig. 4).

The mean difference in latency between rods and cones was $57 \pm 32 \mathrm{~ms}$. This confirmed previously published results of our group [2] and those of other groups [32-34] reporting latency differences in the range of $20 \mathrm{~ms}-100 \mathrm{~ms}$. In our previous paper (applying small focal stimuli) we found a conespecific latency of $277 \pm 25 \mathrm{~ms}$ and a rod-specific latency of $372 \pm 13 \mathrm{~ms}$ [2] which is in accordance with the current results.

Latency differences between photCPC and scotCPC are most probably caused by differences in the retinal processing of the cone and rod system. According to current knowledge, a rod on-stimulation is either transferred from rod bipolar cells via AII amacrine cells to cone bipolar cells or directly via gap junctions from the rod to a cone and thereafter to a cone bipolar cell [35-39]. Consequently, the rod ONpathway includes at least one additional cell compared to the cone ON-pathway.

Latency decreased with increasing radius of circle stimuli for cones and rods (see Fig. 4). The latency difference between the different steps of circle sizes in our study was very similar, no matter how large the change of area was. This indicates a non-linear relation to stimulus size while the relation to the logarithm of stimulus size is negatively linear. These observations are in line with the results of Cibis et al. and $\mathrm{Hu}$ et al.: Cibis et al. found shorter latencies for larger stimuli and additionally a negative linear relation between the logarithm of the stimulus intensity and the pupillomotor latency [40] and $\mathrm{Hu}$ et al. determined latency to be a function of CFD [22]. In ERG, a faster b-wave can be determined with increasing light for both rods and cones [34] which is consistent with our results as well. In contrast, latency remained nearly constant with increasing ring stimulus size for both photoreceptors. This effect indicates that peripheral retinal stimulation might fasten pupillary dynamics leading to a decreased 
latency in comparison to central stimulation. On the other hand, saturation due to stimulus size can be considered.

In conclusion, the main outcome of this study is that the effect of central stimulation on the relMCA is more dominant for cone-specific stimuli than for rodspecific stimuli while latency dynamics are similar for rods and cones despite their absolute latency difference.

The clinical relevance of our study is that smaller stimulus sizes in the range of $3^{\circ}-5^{\circ}$ as used in the CPC or multifocal approaches are necessary to detect central defects under rod-specific test conditions.

We are aware of certain limitations of our study. The pupillary light reaction is a complex process that receives its input not only from rods and cones but also from intrinsically photosensitive retinal ganglion cells (ipRGCs) and will therefore be influenced by diffuse bipolar cells and dopaminergic amacrine cells [29]. Nevertheless, our stimuli were designed to stimulate the photoreceptors as specifically as possible while keeping clinical practicability [2] and being in terms with the ISCEV- and Pupillography-Standards $[29,30]$. Finally, we did predominantly address rods or cones by using different light levels, wavelengths and states of adaptation, but cannot claim an absolute separation of their inputs.

Furthermore, we cannot completely exclude the effect of light scatter. If there was an effect of scatter, it would be expected to be stronger for scotCPC than for photCPC, as rods are more sensitive than cones [41] though a lower stimulus intensity leads to less light scatter. However, as the average retinal diameter of the rod-free zone is only $1.25^{\circ}$ and as there are even more rods than cones at an eccentricity of $3^{\circ}$ according to Curcio et al. [31], it is unlikely that our strong response to the $3^{\circ}$ circle is an effect of scattering. Additionally, none of our probands reported any blurred stimulus margin.

Another point is the effect of refractive errors. Minor refractive errors do not influence pupillary light responses in CPC. However, it is difficult to define a numerical limit. According to our experience from former studies, we believe the limit of $\pm 3 \mathrm{D}$ is reasonable. Because we examine without corrective glasses, higher hyperopia might induce a pupillary near response. In younger subjects, an accommodative near response up to $3 \mathrm{D}$ is very small, in most cases absent [42].
Finally, we evaluated the results of 29 healthy young participants. Whether the same effects apply to other age groups remains open.

\section{Code availability:}

The in-house developed software that was used for pupil measurements is described by Stingl et al. [1] and its code is stored at the Centre for Ophthalmology, University of Tübingen.

Acknowledgements We thank Dr. Anne Kurtenbach for proofreading and helpful advice and Irena Stingl for her graphical support with the figures. Supported by the Egon Schumacher-Stiftung, Barnstorf, Germany, a private foundation without commercial interest. Previous abstract and oral presentation of preliminary results at the 33rd International Pupil Colloquium, 2nd-4th October 2019, Murcia, Spain.

Authors' contributions Conceptualization and Methodology: [Barbara Wilhelm, Krunoslav Stingl, Carina Kelbsch, Anton Sonntag]; Formal analysis and investigation: [Anton Sonntag, Ronja Jung, Krunoslav Stingl]; Data interpretation: [Anton Sonntag, Carina Kelbsch, Ronja Jung, Helmut Wilhelm, Torsten Strasser, Tobias Peters, Krunoslav Stingl, Barbara Wilhelm]; Writing-original draft preparation: [Anton Sonntag]; Writing-review and editing: [Anton Sonntag, Carina Kelbsch, Ronja Jung, Helmut Wilhelm, Torsten Strasser, Tobias Peters, Krunoslav Stingl, Barbara Wilhelm]; All authors read and approved the final manuscript.

Funding Open Access funding enabled and organized by Projekt DEAL. This study was supported by the Egon Schumacher-Stiftung, Barnstorf, Germany, a private foundation without commercial interest.

Availability of data and materials The datasets generated during and/or analysed during the current study are available from the corresponding author on reasonable request.

\section{Declarations}

Conflict of interest The authors have no conflicts of interest to declare that are relevant to the content of this article.

Ethics approval The study was approved by the local ethics committee of the Medical Faculty, University of Tübingen and obeyed the tenets of the Declaration of Helsinki.

Consent to participate All participants received detailed information about the study and its aims and gave their written informed consent to participate.

Consent for publication Patients signed informed consent regarding publishing their data. 
Open Access This article is licensed under a Creative Commons Attribution 4.0 International License, which permits use, sharing, adaptation, distribution and reproduction in any medium or format, as long as you give appropriate credit to the original author(s) and the source, provide a link to the Creative Commons licence, and indicate if changes were made. The images or other third party material in this article are included in the article's Creative Commons licence, unless indicated otherwise in a credit line to the material. If material is not included in the article's Creative Commons licence and your intended use is not permitted by statutory regulation or exceeds the permitted use, you will need to obtain permission directly from the copyright holder. To view a copy of this licence, visit http://creativecommons.org/licenses/by/4.0/.

\section{References}

1. Stingl K, Peters T, Strasser T, Kelbsch C, Richter P, Wilhelm H, Wilhelm B (2018) Pupillographic campimetry: an objective method to measure the visual field. Biomed Tech (Berl) 63(6):729-734. https://doi.org/10.1515/bmt-20170029

2. Kelbsch C, Stingl K, Kempf M, Strasser T, Jung R, Kuehlewein L, Wilhelm H, Peters T, Wilhelm B, Stingl K (2019) Objective measurement of local rod and cone function using gaze-controlled chromatic pupil campimetry in healthy subjects. Transl Vis Sci Technol 8(6):19. https://doi.org/10. 1167/tvst.8.6.19

3. Carle CF, James AC, Kolic M, Essex RW, Maddess T (2015) Blue multifocal pupillographic objective perimetry in glaucoma. Invest Ophthalmol Vis Sci 56(11):6394-6403. https://doi.org/10.1167/iovs.14-16029

4. Najjar RP, Sharma S, Atalay E, Rukmini AV, Sun C, Lock JZ, Baskaran M, Perera SA, Husain R, Lamoureux E, Gooley JJ, Aung T, Milea D (2018) Pupillary responses to full-field chromatic stimuli are reduced in patients with early-stage primary open-angle glaucoma. Ophthalmology 125(9):1362-1371. https://doi.org/10.1016/j.ophtha.2018. 02.024

5. Sabeti F, Maddess T, Essex RW, Saikal A, James AC, Carle CF (2014) Multifocal pupillography in early age-related macular degeneration. Optom Vis Sci 91(8):904-915. https://doi.org/10.1097/opx.0000000000000319

6. Kelbsch C, Lange J, Wilhelm H, Wilhelm B, Peters T, Kempf M, Kuehlewein L, Stingl K (2020) Chromatic pupil campimetry reveals functional defects in exudative age-related macular degeneration with differences related to disease activity. Transl Vis Sci Technol 9(6):5. https://doi.org/ 10.1167/tvst.9.6.5

7. Kardon R, Anderson SC, Damarjian TG, Grace EM, Stone E, Kawasaki A (2011) Chromatic pupillometry in patients with retinitis pigmentosa. Ophthalmology 118(2):376-381. https://doi.org/10.1016/j.ophtha.2010.06.033

8. Kawasaki A, Crippa SV, Kardon R, Leon L, Hamel C (2012) Characterization of pupil responses to blue and red light stimuli in autosomal dominant retinitis pigmentosa due to NR2E3 mutation. Invest Ophthalmol Vis Sci 53(9):5562-5569. https://doi.org/10.1167/iovs.12-10230

9. Chibel R, Sher I, Ben Ner D, Mhajna MO, Achiron A, Hajyahia S, Skaat A, Berchenko Y, Oberman B, KalterLeibovici O, Freedman L, Rotenstreich Y (2016) Chromatic multifocal pupillometer for objective perimetry and diagnosis of patients with Retinitis pigmentosa. Ophthalmology 123(9):1898-1911. https://doi.org/10.1016/j.ophtha.2016. 05.038

10. Stingl KT, Kuehlewein L, Weisschuh N, Biskup S, Cremers FPM, Khan MI, Kelbsch C, Peters T, Ueffing M, Wilhelm B, Zrenner E, Stingl K (2019) Chromatic full-field stimulus threshold and pupillography as functional markers for latestage, early-onset retinitis pigmentosa caused by CRB1 mutations. Transl Vis Sci Technol 8(6):45. https://doi.org/ 10.1167/tvst.8.6.45

11. Sabeti F, Nolan CJ, James AC, Jenkins A, Maddess T (2015) Multifocal pupillography identifies changes in visual sensitivity according to severity of diabetic retinopathy in type 2 diabetes. Invest Ophthalmol Vis Sci 56(8):4504-4513. https://doi.org/10.1167/iovs.15-16712

12. Kardon R, Anderson SC, Damarjian TG, Grace EM, Stone E, Kawasaki A (2009) Chromatic pupil responses: preferential activation of the melanopsin-mediated versus outer photoreceptor-mediated pupil light reflex. Ophthalmology 116(8):1564-1573. https://doi.org/10.1016/j.ophtha.2009. 02.007

13. Kelbsch C, Maeda F, Lisowska J, Lisowski L, Strasser T, Stingl K, Wilhelm B, Wilhelm H, Peters T (2017) Analysis of retinal function using chromatic pupillography in retinitis pigmentosa and the relationship to electrically evoked phosphene thresholds. Acta Ophthalmol 95(4):e261-e269. https://doi.org/10.1111/aos.13259

14. Lisowska J, Lisowski L, Kelbsch C, Maeda F, Richter P, Kohl S, Zobor D, Strasser T, Stingl K, Zrenner E, Peters T, Wilhelm H, Fischer MD, Wilhelm B (2017) Development of a chromatic pupillography protocol for the first gene therapy trial in patients with CNGA3-linked achromatopsia. Invest Ophthalmol Vis Sci 58(2):1274-1282. https://doi. org/10.1167/iovs.16-20505

15. Kelbsch C, Maeda F, Strasser T, Blumenstock G, Wilhelm B, Wilhelm H, Peters T (2016) Pupillary responses driven by ipRGCs and classical photoreceptors are impaired in glaucoma. Graefes Arch Clin Exp Ophthalmol 254(7):1361-1370. https://doi.org/10.1007/s00417-0163351-9

16. Skorkovská K, Wilhelm H, Ludtke H, Wilhelm B, Kurtenbach A (2014) Investigation of summation mechanisms in the pupillomotor system. Graefes Arch Clin Exp Ophthalmol 252(7):1155-1160. https://doi.org/10.1007/s00417014-2677-4

17. Loewenfeld IE (1993) The light reflex. The pupil: anatomy, physiology and clinical applications, vol I. Wayne State University Press, Detroit, pp 83-273

18. Kardon RH (1992) Pupil perimetry. Curr Opin Ophthalmol 3(5):565-570. 199210000-00002

19. Kardon RH, Kirkali PA, Thompson HS (1991) Automated pupil perimetry: Pupil field mapping in patients and normal subjects. Ophthalmology 98(4):485-495. https://doi.org/10. 1016/s0161-6420(91)32267-x 
20. Stanley PA, Davies AK (1995) The effect of field of view size on steady-state pupil diameter. Ophthalm Physiol Opt 15(6):601-603. https://doi.org/10.1046/j.1475-1313.1995. 9400019v.x

21. Watson AB, Yellott JI (2012) A unified formula for lightadapted pupil size. J Vis 12(10):1-16. https://doi.org/10. $1167 / 12.10 .12$

22. Hu X, Hisakata R, Kaneko H (2020) Effects of stimulus size, eccentricity, luminance, and attention on pupillary light response examined by concentric stimulus. Vis Res 170:35-45. https://doi.org/10.1016/j.visres.2020.03.008

23. Park JC, McAnany JJ (2015) Effect of stimulus size and luminance on the rod-, cone-, and melanopsin-mediated pupillary light reflex. J Vis 15(3):1-13. https://doi.org/10. $1167 / 15.3 .13$

24. Schmid R, Wilhelm B, Wilhelm H (2000) Pupillomotor campimetry in normals. Neuroophthalmology 23(1):7-13. https://doi.org/10.1076/0165-8107(200002)2311-DFT007

25. Haj Yahia S, Hamburg A, Sher I, Ben Ner D, Yassin S, Chibel R, Mimouni M, Derazne E, Belkin M, Rotenstreich Y (2018) Effect of stimulus intensity and visual field location on rod- and cone-mediated pupil response to focal light stimuli. Invest Ophthalmol Vis Sci 59(15):6027-6035. https://doi.org/10.1167/iovs.18-23767

26. Joyce DS, Feigl B, Zele AJ (2016) Melanopsin-mediated post-illumination pupil response in the peripheral retina. J Vis 16(8):1-15. https://doi.org/10.1167/16.8.5

27. Li D, Winfield D, Parkhurst DJ (2005) Starburst: a hybrid algorithm for video-based eye tracking combining featurebased and model-based approaches. In: Proceedings of the 2005 IEEE computer society conference on computer vision and pattern recognition (CVPR'05)—workshops, vol 3. IEEE Computer Society, p 79. https://doi.org/10.1109/cvpr. 2005.531

28. Fischler MA, Bolles RC (1981) Random sample consensus: a paradigm for model fitting with applications to image analysis and automated cartography. Commun ACM 24(6):381-395. https://doi.org/10.1145/358669.358692

29. Kelbsch C, Strasser T, Chen Y, Feigl B, Gamlin PD, Kardon R, Peters T, Roecklein KA, Steinhauer SR, Szabadi E, Zele AJ, Wilhelm H, Wilhelm BJ (2019) Standards in pupillography. Front Neurol 10:129. https://doi.org/10.3389/fneur. 2019.00129

30. McCulloch DL, Marmor MF, Brigell MG, Hamilton R, Holder GE, Tzekov R, Bach M (2015) ISCEV Standard for full-field clinical electroretinography (2015 update). Doc Ophthalmol 130(1):1-12. https://doi.org/10.1007/s10633014-9473-7
31. Curcio CA, Sloan KR, Kalina RE, Hendrickson AE (1990) Human photoreceptor topography. J Comp Neurol 292(4):497-523. https://doi.org/10.1002/cne.902920402

32. Barbur JL (1982) Reaction-time determination of the latency between visual signals generated by rods and cones. Ophthalm Physiol Opt 2(3):179-185. https://doi.org/10. 1111/j.1475-1313.1982.tb00175.x

33. Cao D, Zele AJ, Pokorny J (2007) Linking impulse response functions to reaction time: rod and cone reaction time data and a computational model. Vis Res 47(8):1060-1074. https://doi.org/10.1016/j.visres.2006.11.027

34. Zrenner E, Holder GE, Schiefer U, Wild JM (2020) quality control procedures and baseline values for electroretinography, perimetry, color vision, and visual acuity in an international multicenter study: observations from a safety trial in chronic stable angina pectoris. Transl Vis Sci Technol 9(8):38. https://doi.org/10.1167/tvst.9.8.38

35. Smith RG, Freed MA, Sterling P (1986) Microcircuitry of the dark-adapted cat retina: functional architecture of the rod-cone network. J Neurosci 6(12):3505-3517. https://doi. org/10.1523/jneurosci.06-12-03505.1986

36. DeVries SH, Baylor DA (1995) An alternative pathway for signal flow from rod photoreceptors to ganglion cells in mammalian retina. Proc Natl Acad Sci USA 92(23):10658-10662. https://doi.org/10.1073/pnas.92.23. 10658

37. Famiglietti EV, Kolb H (1975) A bistratified amacrine cell and synaptic circuitry in the inner plexiform layer of the retina. Brain Res 84(2):293-300. https://doi.org/10.1016/ 0006-8993(75)90983-x

38. Raviola E, Dacheux RF (1987) Excitatory dyad synapse in rabbit retina. Proc Natl Acad Sci USA 84(20):7324-7328. https://doi.org/10.1073/pnas.84.20.7324

39. Demb JB, Pugh EN (2002) Connexin 36 forms synapses essential for night vision. Neuron 36(4):551-553. https:// doi.org/10.1016/s0896-6273(02)01062-0

40. Cibis GW, Campos EC, Aulhorn E (1977) Pupillomotor latent period. Vis Res 17(6):737-738. https://doi.org/10. 1016/s0042-6989(77)80012-6

41. Ingram NT, Sampath AP, Fain GL (2016) Why are rods more sensitive than cones? J Physiol 594(19):5415-5426. https://doi.org/10.1113/jp272556

42. Wilhelm H, Schaeffel F, Wilhelm B (1993) Age dependence of pupillary near reflex. Klin Monbl Augenheilkd 203(2):110-116. https://doi.org/10.1055/s-2008-1045657

Publisher's Note Springer Nature remains neutral with regard to jurisdictional claims in published maps and institutional affiliations. 